\title{
Advances in HER2-Positive Breast Cancer: Novel Therapies and Adverse Event Management
}

A continuing education activity for nurse practitioners, PAs, clinical nurse specialists, advanced degree nurses, oncology and hematology nurses, pharmacists, and physicians.

\section{Harborside Medical Education}

Harborside Medical Education (HSME), a division of Harborside, is committed to advancing care for people with cancer and improving patient outcomes through professional continuing education (CE) activities in oncology that address practice gaps and promote change in learners' knowledge, competence and performance in both community and academic practice settings.

Release date: March 21, 2019

Estimated time to complete activity: 0.5 hour

Expiration date: March 21, 2020

Certified for CE by:

Annenberg Center for Health Sciences at Eisenhower

39000 Bob Hope Drive, Dinah Shore Bldg.

Rancho Mirage, CA 92270

Voice: $760-773-4500$

Fax: 760-773-4513

E-mail: contactce@annenberg.net

(C) 2019, Annenberg Center for Health Sciences

at Eisenhower. All rights reserved.

\author{
Journal of the Advanced Practitioner \\ in Oncology \\ 94 North Woodhull Road \\ Huntington, NY 11743 \\ Voice: 631-692-0800 \\ Fax: 631-692-0805 \\ E-mail: editor@advancedpractitioner.com
}

This certified activity is supported by an educational grant from Puma Biotechnology, Inc.

\section{Faculty}

Reshma Mahtani, DO, Sylvester Comprehensive Cancer Center, Miami, Florida

Lisa Hineman, MS, AOCN ${ }^{\circledR}$, PHN, ANP-C, Los Angeles Cancer Network, Los Angeles, California

\section{Intended Audience}

The activity's target audience will consist of nurse practitioners, physician assistants, clinical nurse specialists, advanced degree nurses, oncology and hematology nurses, pharmacists, and physicians.

\section{Learning Objectives}

After completing this educational activity, participants should be able to:

1. Discuss the clinical significance of existing and emerging data in HER2+ breast cancer

2. Develop a strategy to effectively manage diarrhea and other adverse events associated with treatments for HER2+ breast cancer

3. Implement best practices for the management of HER2+ breast cancer brain metastases 


\section{Continuing Education}

Statement of Credit-Participants who successfully complete this activity (including the submission of the post-test and evaluation form) will receive a statement of credit.

Physicians. This activity has been planned and implemented in accordance with the accreditation requirements and policies of the Accreditation Council for Continuing Medical Education through the joint providership of the Annenberg Center for Health Sciences at Eisenhower and the Journal of the Advanced Practitioner in Oncology. The Annenberg Center is accredited by the ACCME to provide continuing medical education for physicians.

The Annenberg Center for Health Sciences at Eisenhower designates this enduring activity for a maximum of 0.5 AMA PRA Category 1 Credits $^{\mathrm{TM}}$. Physicians should claim only the credit commensurate with the extent of their participation in the activity.

Nurses. The Annenberg Center for Health Sciences at Eisenhower is accredited as a provider of continuing nursing education by the American Nurses Credentialing Center's Commission on Accreditation.

A maximum of 0.5 contact hours may be earned for successful completion of this activity.

Provider approved by the California Board of Registered Nursing, Provider No. 13664, for 0.5 contact hours.

AG

Pharmacists. The Annenberg Center for Health Sciences at Eisenhower is accredited by the Accreditation Council for Pharmacy Education (ACPE) as a provider of continuing pharmacy education. This program has been assigned ACPE Universal Program \#0797-9999-19-041-H01-P. This program is designated for up to 0.5 contact hours (0.05 CEUs) of continuing pharmacy education credit.

\section{Financial Disclosures}

All individuals in positions to control the content of this program (e.g., planners, faculty, content reviewers) are expected to disclose all financial relationships with commercial interests that may have a direct bearing on the subject matter of this continuing education activity. Annenberg Center for Health Sciences at Eisenhower has identified and resolved all conflicts of interest in accordance with the ACHS policies and procedures. Participants have the responsibility to assess the impact (if any) of the disclosed information on the educational value of the activity.

\section{Disclosures}

\section{FACULTY}

Dr. Mahtani has consulted for Amgen, Genentech, Lilly, Novartis, Pfizer, and Puma, and has received research support from Genentech.

Ms. Hineman has nothing to disclose.

\section{ANNENBERG CENTER FOR HEALTH SCIENCES AT EISENHOWER}

Staff at the Annenberg Center for Health Sciences at Eisenhower have no relevant commercial relationships to disclose.

\section{LEAD NURSE PLANNER}

Dorothy Caputo, MA, BSN, RN, EduPro Resources, LLC, has nothing to disclose.

\section{PLANNERS}

Harborside and Harborside Medical Education staff have no potential conflicts of interest to disclose. 


\section{CONTENT REVIEWERS}

Monique Johnson, MD, CHCP, has nothing to disclose.

Kelley Mayden, MSN, FNP, AOCNP ${ }^{\circledR}$, has served on speakers bureaus for Pfizer and Puma.

Wendy Vogel, MSN, FNP, AOCNP ${ }^{\circledR}$, has served on speakers bureaus for AMAG, Celgene, Genentech, Ipsen, Lilly, and Novartis.

\section{ARTICLE COPYWRITER}

Barry A. Fiedel, PhD, OnPoint Medical Communications LLC, has nothing to disclose.

\section{Disclaimer}

This activity has been designed to provide continuing education that is focused on specific objectives. In selecting educational activities, clinicians should pay special attention to the relevance of those objectives and the application to their particular needs. The intent of all Annenberg Center for Health Sciences at Eisenhower educational opportunities is to provide learning that will improve patient care. Clinicians are encouraged to reflect on this activity and its applicability to their own patient population.

The opinions expressed in this activity are those of the faculty and reviewers and do not represent an endorsement by Annenberg Center for Health Sciences at Eisenhower of any specific therapeutics or approaches to diagnosis or patient management.

\section{Product Disclosure}

This educational activity may contain discussion of published as well as investigational uses of agents that are not approved by the US Food and Drug Administration. For additional information about approved uses, including approved indications, contraindications, and warnings, please refer to the prescribing information for each product.

\section{How to Earn Credit}

To access the learning assessment and evaluation form online, visit https://education.annenberg.net/jadproher2article

Statement of Credit: Participants who successfully complete this activity (including scoring of a minimum of $70 \%$ on the learning assessment) and complete and submit the evaluation form will be able to download a statement of credit. 


\title{
Advances in HER 2-Positive Breast Cancer: Novel Therapies and Adverse Event Management
}

\author{
RESHMA MAHTANI, DO, and LISA HINEMAN, MS, AOCN ${ }^{\circledR}$, PHN, ANP-C
}

From Sylvester Comprehensive Cancer Center, University of Miami Health System, Miami, Florida, and Los Angeles Cancer Network, Los Angeles, California

Authors' disclosures of conflicts of interest are found at the end of this article.

Correspondence to: Reshma Mahtani, DO, University of Miami Health System, 1192 East Newport Center Drive, Deerfield Beach, FL 33442. E-mail: rmahtani@miami.edu.

https://doi.org/10.6004/jadpro.2019.10.2.4 (c) 2019 Harborside $^{\mathrm{TM}}$

\begin{abstract}
Although breast cancer is a heterogeneous disease, approximately $20 \%$ to $25 \%$ of patients diagnosed with breast cancer have amplification of the HER2 gene. The FDA approval of trastuzumab for the treatment of HER2-positive (HER2+) metastatic breast cancer in 1998 represented a major breakthrough for patients with HER2+ disease. In 2006, the FDA extended its approval for use in the adjuvant setting. In recent years, trials have been conducted to identify the appropriate duration of therapy in combination with chemotherapy. More recently, trials incorporating newer HER2-targeted therapies have been conducted. Some trials have demonstrated the importance of considering neoadjuvant HER2-directed therapies for selected patients with HER2+ disease, highlighting the fact that achieving pathologic complete response has important prognostic implications. In order to continue treatment and optimize patient safety, the effective and timely management of treatment-related adverse events (AEs) is crucial. As they are on the front lines of patient care, advanced practitioners need to be able to assess the clinical implications of recent advances and integrate them into practice. One area of unmet need in the management of metastatic HER2-positive disease is the treatment of brain metastases, with several promising therapies under investigation. Using several case studies as a foundation, this article highlights current and emerging data on HER2-directed therapies, outlines strategies for managing AEs, and reviews the key issues surrounding brain metastases and associated novel therapies under investigation.
\end{abstract}

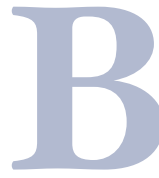

ased on data from the American Cancer Society (ACS), survival is improving for patients with breast cancer (BC), largely because of earlier detection and improved treatment modalities (ACS, 2018).
Notwithstanding these improvements, nearly 42,000 women died from metastatic breast cancer (MBC) in 2018 (ACS, 2018).

Breast cancer is a heterogeneous disease; approximately $20 \%$ to $25 \%$ of BCs have amplification 
of the human epidermal growth factor receptor 2 (HER2) gene. This gene was discovered more than 30 years ago, and at the time, conferred a poor prognosis. HER $2+\mathrm{BC}$ is common in younger patients and is associated with poorly differentiated, high-grade tumors (Burstein, 2005); approximately 35,000 cases are diagnosed annually (Howlader et al., 2014). A growing understanding of the pathobiology of HER2+ BC led to the development and approval of trastuzumab, which has resulted in a substantial change in the natural history of this cancer subtype (Slamon et al., 2011, 2015). The approval of trastuzumab for the treatment of HER2+ MBC in 1998 represented a major breakthrough for patients with HER2+ disease. In 2006, trastuzumab received US Food and Drug Administration (FDA) approval for use in the adjuvant setting.

Since then, the therapeutic landscape has continued to evolve. Trials have been conducted to identify the appropriate duration of therapy in combination with chemotherapy. More recently, results of trials incorporating newer HER2targeted therapies, either given as dual blockade or sequentially following standard trastuzumab adjuvant therapy, have been reported. Several studies have demonstrated the importance of considering neoadjuvant HER2-directed therapies for certain patients with HER2+ disease, with the understanding that achievement of a pathologic complete response ( $\mathrm{pCR}$ ) has important prognostic implications.

Best practices for the management of treatment-related adverse events (AEs) are evolving as well. One area of unmet need in the management of metastatic HER2+ disease is the treatment of brain metastases, with several new and exciting therapies under investigation. To provide optimal patient-centered care, advanced practitioners (APs) need to be able to assess the clinical implications of all of these advances and appropriately integrate them into practice.

\section{Case 1: Adjuvant and Extended Adjuvant Therapy}

The first case is that of a 45-year-old woman with no prior relevant medical history. A mass was palpated in her left breast, biopsy-proven to be estrogen receptor-negative (ER-), progesterone receptor-negative (PR-), and HER2+ (3+ by immunohistochemistry [IHC]). Lymph nodes were suggestive of metastases on imaging and were biopsied and determined to be positive. The patient was staged as cT1cN1MO. A lumpectomy was performed, with final pathology documenting a 1.8$\mathrm{cm}$ invasive ductal carcinoma, with 3 of 7 nodes positive. The patient received docetaxel/carboplatin/trastuzumab/pertuzumab (TCHP) for 6 cycles following surgery. Following this, she completed 1 year of adjuvant trastuzumab and pertuzumab, as well as post-lumpectomy radiation.

\section{ADJUVANT SYSTEMIC THERAPY}

Case 1 highlights the appropriate use of adjuvant chemotherapy and HER2-directed therapies following surgery. While there are data to support the use of anthracycline as well as nonanthracycline regimens in the adjuvant setting, HER2+ disease is aggressive, and therefore most patients with early-stage BC will require adjuvant chemotherapy and trastuzumab (the exception being patients with TlaNo tumors, for which the data are less clear). Four large, randomized adjuvant trastuzumab clinical trials have shown that the addition of 1 year of trastuzumab not only improves disease-free survival (DFS) but also overall survival (OS) in patients with HER2+ BC. These four landmark trials include HERA, NSABP B-31, NCCTG N9831, and BCIRG 006. Their respective study designs are shown in Figure 1 (Goldhirsch et al., 2013; Perez et al., 2014; Romond et al., 2005; Slamon et al., 2015).

\section{Dual Anti-HER2 Adjuvant Therapy}

Despite improvements in efficacy associated with the use of adjuvant trastuzumab, there is still room for improvement, as shown in Figure 2, which demonstrates long-term outcomes from BCIRG-006 and NSABP B-31/NCCTG N9831. The graphs illustrate that 1 year of adjuvant trastuzumab does not eliminate the risk of recurrence for all patients with HER2+ BC (Perez et al., 2014; Slamon et al., 2015).

\section{Improving on 1 Year of Adjuvant Trastuzumab: APHINITY}

For women with HER2+ early-stage BC who are at high risk of recurrence, additional HER2-targeted 


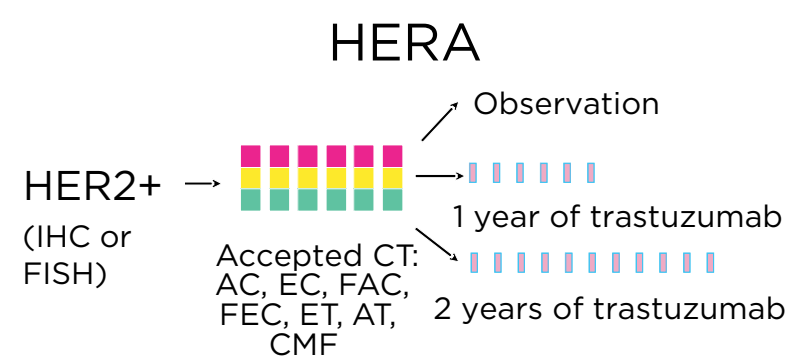

NCCTG N9831

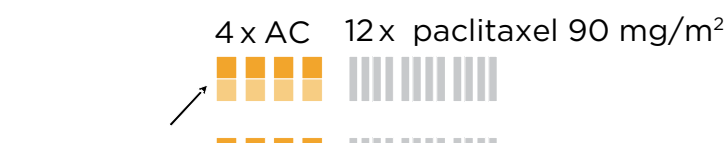

HER2+ $\rightarrow$ -

(IHC or FISH)

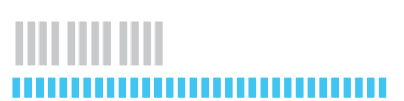

1 year of trastuzumab

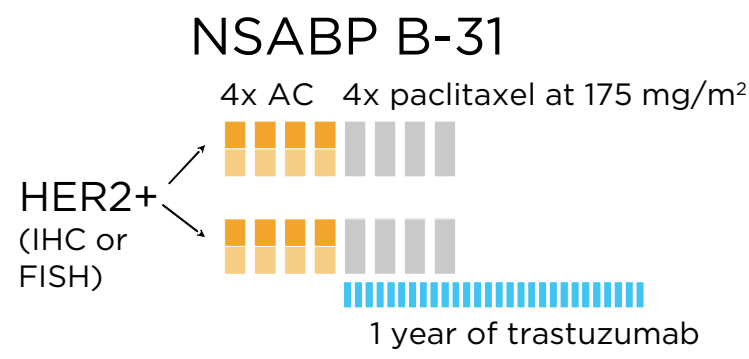

BCIRG 006

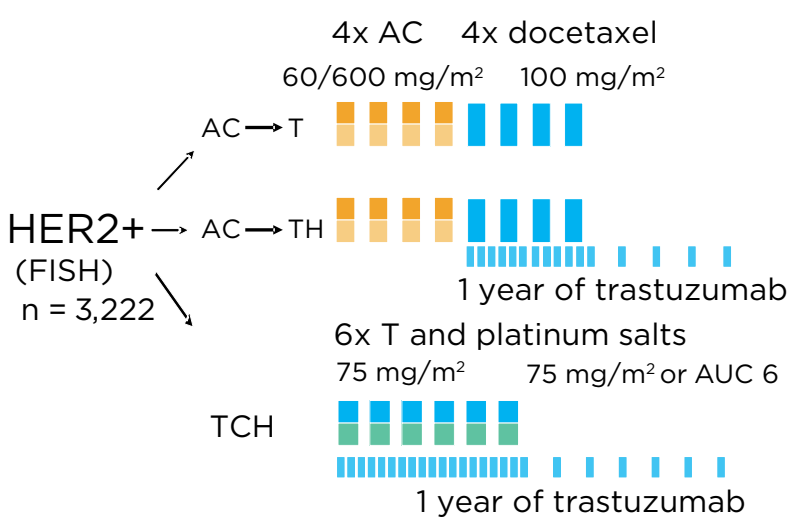

Figure 1. Landmark adjuvant trials; 4 positive adjuvant trastuzumab trials. AC = doxorubicin/cyclophosphamide; $A T$ = doxorubicin/docetaxel; $A U C=$ area under the curve; $C M F=$ cyclophosphamide/ methotrexate/fluorouracil; $C T$ = chemotherapy; $E C$ = epirubicin/cyclophosphamide; $E T$ = epirubicin/ docetaxel; FAC = fluorouracil/doxorubicin/cyclophosphamide; FEC = fluorouracil/epirubicin/cyclophosphamide; FISH = fluorescence in situ hybridization; HER2 = human epidermal growth factor receptor 2; IHC = immunohistochemistry; T = docetaxel; TCH = docetaxel/carboplatin/trastuzumab; $\mathrm{TH}=$ docetaxel/trastuzumab. Adapted from Goldhirsch et al. (2013); Perez et al. (2014); Romond et al. (2005); Slamon et al. (2011).

therapy may be indicated, as demonstrated by APHINITY, a phase III randomized clinical trial of 4,805 patients with operable HER2+ BC that evaluated the efficacy and safety of pertuzumab plus trastuzumab and chemotherapy compared with those of trastuzumab and chemotherapy in the adjuvant setting (von Minckwitz et al., 2017). Sixty-three percent of patients were node-positive and $36 \%$ of patients had HR-negative disease. Patients who had tumors between 0.5 and $1 \mathrm{~cm}$ were allowed to enroll if they had one high-risk feature (age $<35$, grade 3 , or ER- and PR- status). The study design is shown in Figure 3.

At the 4-year mark, a 1.7\% benefit was seen for patients receiving adjuvant pertuzumab in combination with trastuzumab compared to that in patients who received trastuzumab alone, a small but significant finding (Figure 4). The magnitude of benefit was greater in node-positive and HRnegative disease. In regard to safety with the addition of pertuzumab, there were no specific additional safety signals, including any meaningful cardiac toxicity (Table 1; von Minckwitz et al., 2017).

APHINITY demonstrated that adjuvant pertuzumab plus trastuzumab plus cytotoxic chemotherapy significantly reduced the risk of recurrence compared with that of placebo plus trastuzumab plus chemotherapy in patients with HER2+ early BC, supporting the approach to treatment for the patient in case 1 , who presented with high-risk disease (node positivity and ER- status).

Another consideration for the patient in case 1 (especially if her tumor had been ER+ as opposed to ER-) is the use of extended adjuvant therapy with neratinib, an oral, irreversible tyrosine kinase inhibitor that demonstrated efficacy in patients 

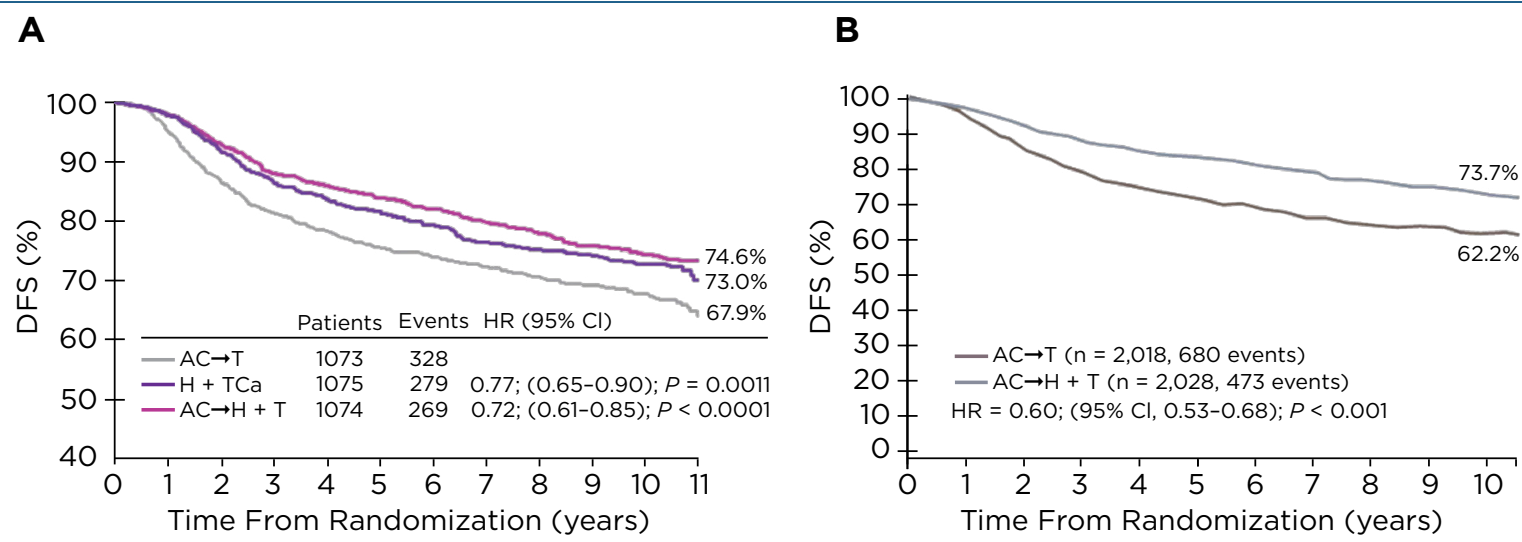

Figure 2. Long-term data in patients who were treated with adjuvant chemotherapy with or without trastuzumab for 1 year. (A) BCIRG-006 long-term follow-up: disease-free survival. (B) NSABP B-31 and NCCTG N9831 joint analysis. Despite one year of adjuvant tratment, HER2+ patients still experienced recurrence. $\mathrm{AC}=$ doxorubicin and cyclophosphamide; $\mathrm{BCIRG}=$ Breast Cancer International Research Group; $\mathrm{Ca}=$ carboplatin; $\mathrm{Cl}$ = confidence interval; $\mathrm{DFS}=$ disease-free survival; $\mathrm{H}=$ trastuzumab; $\mathrm{HR}=$ hazard ratio; NSABP = National Surgical Adjuvant Breast and Bowel Project; NCCTG $=$ North Central Cancer Treatment Group; T = taxane. Adapted from Perez et al. (2014); Slamon et al. (2015).

with ER+/HER2+ disease, as well as in the intention-to-treat population, at high risk for relapse in the ExteNET trial.

\section{Extended Adjuvant Benefit: ExteNET}

ExteNET enrolled 2,840 patients with HER2+ BC, stage I to III, who had completed adjuvant trastuzumab for 1 year into a randomized, double-blind, placebo-controlled, phase III trial. Patients were subsequently randomized to 1 additional year of neratinib or placebo (Figure 5; Chan et al., 2016; Martin et al., 2017).

Neratinib inhibits HER1, HER2, and HER4 and binds to the intercellular signaling domain of HER1, HER2, HER3, and epithelial growth factor receptor; binding inhibits phosphorylation and several HER downstream signaling pathways, which leads to decreased proliferation and increased cell death. The 3-year invasive disease-free survival (iDFS) was $92 \%$ for neratinib vs. $89.9 \%$ for placebo (Chan et al., 2016; Martin et al., 2017). Results were updated in 2017, wherein the 5-year iDFS was $90.2 \%$ with neratinib vs. $87.7 \%$ with placebo, an overall benefit of about 2.5\% (Figure 6; Martin et al., 2017). When findings were analyzed by receptor status, benefit was greatest in the $\mathrm{HR}+$ patients (absolute $4.4 \%$ iDFS benefit; hazard ratio of $0.60 ; 95 \%$
$\mathrm{CI}=0.43-0.83$; Figure 7; Martin et al., 2017). The authors suggested that neratinib may modulate ER sensitivity to hormonal therapy, resulting in bidirectional crosstalk.

ExteNET conclusions were that neratinib provides sustained benefit for women with early-stage, HER2+ BC following adjuvant trastuzumab, with an overall benefit of $2.5 \%$ and an absolute benefit of $5.1 \%$ in HR+ patients. No indications of long-term toxicity have emerged (cardiotoxicity or secondary malignancies). The main AE was grade 3 diarrhea, which occurred in $40 \%$ of patients, most of which resolved in fewer than 30 days (1.4\% of patients required hospitalization). Patients reported a transient reduction in health-related quality of life during the first month of therapy, followed by steady recovery toward baseline. The follow-up CONTROL trial (Hurvitz et al., 2017) showed the incidence of diarrhea to be further reduced as compared to the ExteNET trial with the use of prophylactic loperamide alone or in combination with budesonide, or colestipol.

The patient in case 1 was treated with dual antibody therapy and, as such, was not represented in the ExteNET trial. In addition, because this patient had ER- disease, there would be less enthusiasm to offer her extended adjuvant treat- 
- International, randomized, double-blind, placebo-controlled phase III trial

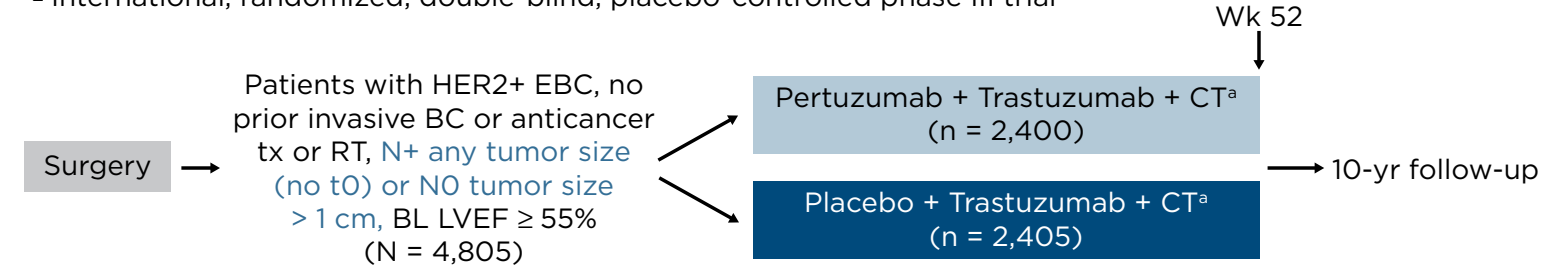

Or node negative with tumors $>0.5$ to $\leq 1 \mathrm{~cm}+$ at least 1 of following: histologic/nuclear grade 3; ER negative and PgR negative; aged < 35 yr. Node-negative enrollment capped after first 3,655 patients randomized.

- Primary endpoint: IDFS per modified STEEP definition (excludes second primary non-BC as event).

- Secondary endpoints: IDFS per STEEP definition. OS = distant recurrance-free survival; DFS = recurrance-free interval, safety, cardiac safety, health-related QoL.

Figure 3. APHINITY study design. BC = breast cancer; $B L=$ baseline; $C T$ = chemotherapy; DFS = disease-free survival; $E B C=$ early breast cancer; HR = hormone receptor; IDFS = invasive disease-free survival; LVEF = left ventricular ejection fraction; QoL = quality of life; RT = radiotherapy; tx = treatment. Adapted from Hudis et al. (2007); von Minckwitz et al. (2017).

aTreatment initiated $\leq 8$ weeks post-surgery. Permitted CT: standard anthracycline or nonanthracycline regimens. Endocrine and/or radiotherapy could be started at end of adjuvant CT.

ment with neratinib, although there are data from an exploratory analysis that demonstrated patients with ER-/HER2+ disease who were randomized to receive neratinib within 6 months of completion of trastuzumab demonstrated a $2.8 \%$ benefit (Martin et al., 2017).

\section{Key Points}

- The addition of 1 year of adjuvant trastuzumab improves not only DFS but also OS in patients with early-stage disease

- Adjuvant pertuzumab plus trastuzumab plus cytotoxic chemotherapy significantly

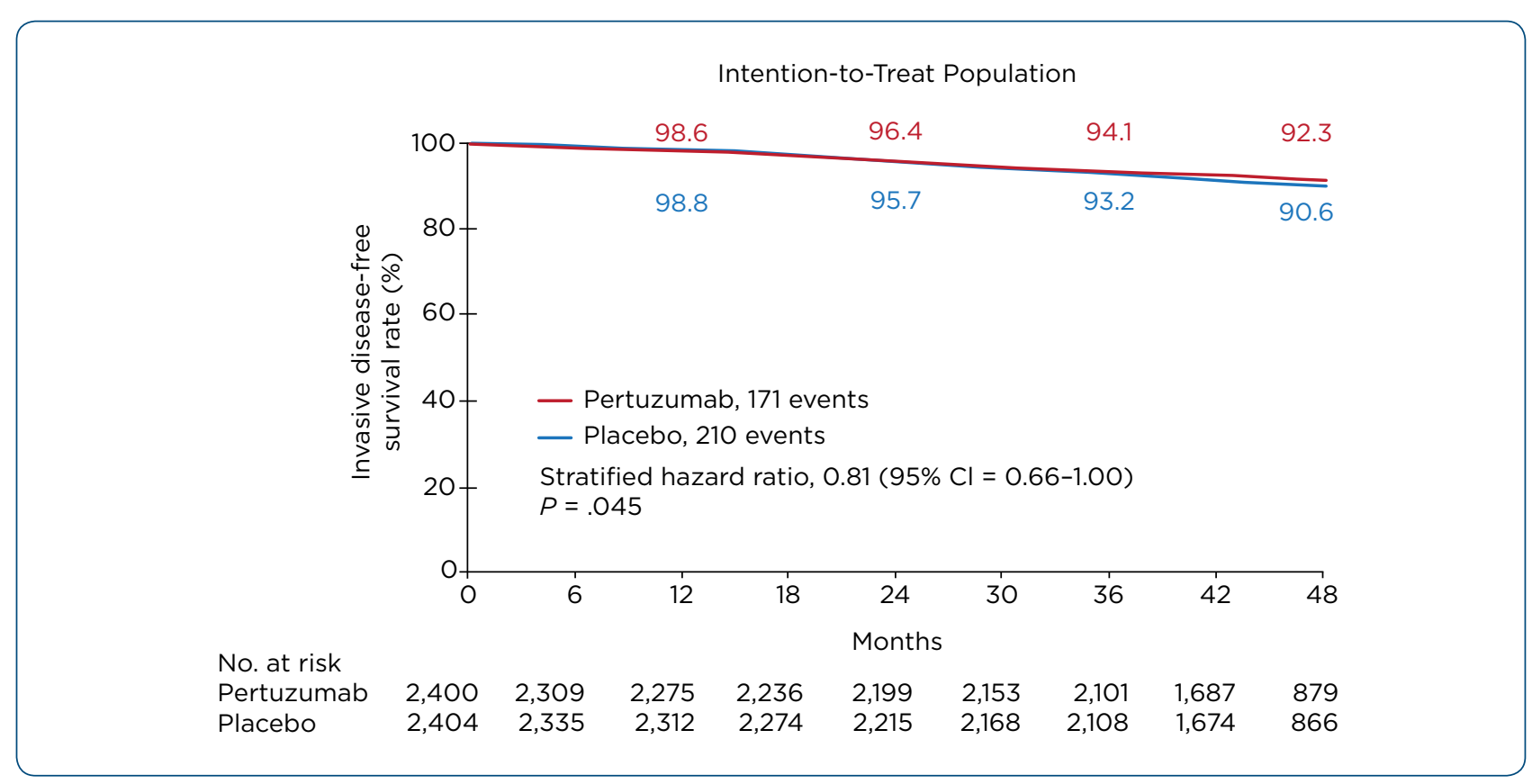

Figure 4. APHINITY interim analysis of invasive disease-free survival (intention to treat). $f / u=f o l l o w-u p ;$ IDFS = invasive disease-free survival; ITT = intention to treat. Data cutoff after 381 IDFS events (median f/u: $45.4 \mathrm{mo}$ ). Adapted from von Minckwitz et al. (2017). 


\begin{tabular}{|c|c|c|c|}
\hline Outcome, n (\%) & $\begin{array}{l}\text { Pertuzumab } \\
(n=2,364)\end{array}$ & $\begin{array}{l}\text { Placebo } \\
(n=2,405)\end{array}$ & $\begin{array}{l}\text { Treatment difference } \\
(95 \% \mathrm{CI})\end{array}$ \\
\hline Primary cardiac endpoint & $17(0.7)$ & $8(0.3)$ & $0.4 \%(0 \%$ to $0.8 \%)$ \\
\hline Heart failure NYHA III/IV + LVEF drop ${ }^{a}$ & $15(0.6)$ & $6(0.2)$ & \\
\hline Cardiac death ${ }^{b}$ & $2(0.1)$ & $2(0.1)$ & \\
\hline Recovered according to LVEF & $7(0.3)$ & $4(0.2)$ & \\
\hline Asymptomatic or mildly symptomatic LVEF drop ${ }^{c}$ & $64(2.7)$ & $67(2.8)$ & $-0.1 \%(-1.0 \%$ to $0.9 \%)$ \\
\hline \multicolumn{4}{|l|}{ Grade $\geq 3$ AEs } \\
\hline Neutropenia & 16.3 & 15.7 & \\
\hline Febrile neutropenia & 12.1 & 11.1 & \\
\hline Decreased neutrophil count & 9.6 & 9.6 & \\
\hline Diarrhea & 9.8 & 3.7 & \\
\hline Anthracycline $\mathrm{CT}, \mathrm{n} / \mathrm{N}(\%)$ & 7.5 & 3.1 & \\
\hline Nonanthracycline CT, n/N (\%) & 18.0 & 6.1 & \\
\hline Anemia & 6.9 & 4.7 & \\
\hline Fatal AE & $18(0.8)$ & $20(0.8)$ & \\
\hline \multicolumn{4}{|c|}{$\begin{array}{l}\text { Note. Adapted from von Minckwitz et al. (2017). } \\
\text { aLVEF drop defined as ejection fraction decrease } \geq 10 \% \text { from baseline to below 50\%. } \\
\text { bDetermined by Cardiac Advisory Board per prospective definition. } \\
\text { 'Secondary cardiac endpoint. }\end{array}$} \\
\hline
\end{tabular}

reduces the risk of recurrence in patients with high-risk disease

- Neratinib further improves iDFS in the extended adjuvant setting after 1 year of standard adjuvant trastuzumab with no evidence of long-term toxicity

\section{Case 2: Treatment for Residual Disease Following Neoadjuvant Therapy}

The patient is a 65 -year-old woman with no prior relevant medical history. A mass palpated in her left breast was approximately $3 \mathrm{~cm}$ by imaging without abnormal appearing nodes, and biopsy- proven to be invasive ductal cancer, ER+, HER2+ (3+ by IHC), cT2NOM0. She received neoadjuvant TCHP $\times 6$ cycles and did not achieve a pCR at the time of lumpectomy and sentinel lymph node biopsy. Residual disease was noted: $1 \mathrm{~cm}$ in the breast without involvement in the sentinel lymph nodes. She went on to receive post-lumpectomy radiation and receives hormonal therapy with an aromatase inhibitor in the adjuvant setting.

\section{NEOADJUVANT THERAPY}

Patients with a high tumor-volume-to-breast ratio are not candidates for breast conservation. Pa-

\footnotetext{
Stratified by hormone receptor status (ER+ and/or PgR+ vs. ER- and PgR-), nodal status ( $O$ vs. $1-3$ vs. $\geq 4$ ), adjuvant trastuzumab regimen (sequential vs. concurrent with $\mathrm{CT}$ ) $\downarrow$

Patients with HER2+ EBC (stage I-III); adjuvant trastuzumab completed $\leq 2 \mathrm{yr}$ before randomization*; $\mathrm{N}+/$ - disease or residual disease after neoadjuvant therapy; known ER and PgR status $(N=2,840)$

- Primary endpoint: IDFS at 2 yr

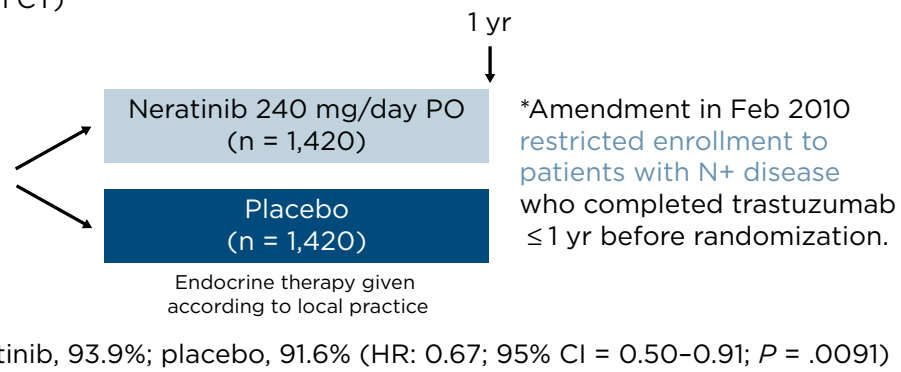

Figure 5. ExteNET 5-year update: Neratinib vs. PBO after adjuvant trastuzumab in HER2+ EBC. CT = chemotherapy; EBC = early breast cancer; IDFS = invasive disease-free survival; $\mathrm{PBO}=\mathrm{placebo}$; $\mathrm{pCR}=$ pathologic CR; PgR = progesterone receptor. Adapted from Chan et al. (2016); Martin et al. (2017). 


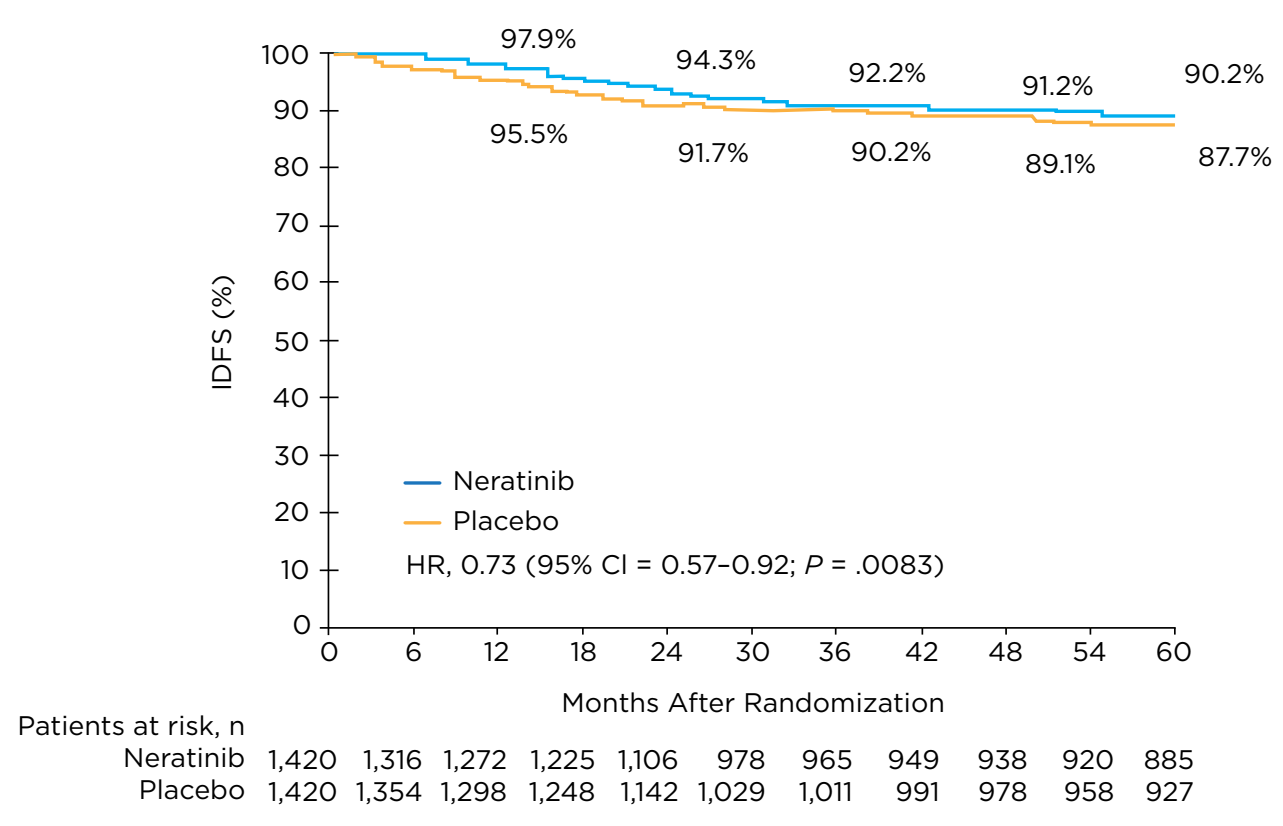

Figure 6. ExteNET: 5-year invasive disease-free survival analysis. Adapted from Martin et al. (2017).

tients with lymph node-positive disease, high-risk features (high grade, ER-, HER2+, or triple-negative disease), and/or younger age at diagnosis are generally considered for neoadjuvant therapy in the early-stage setting. More specifically, patients with HER2+, T2 or greater, and/or node-positive disease should be offered chemotherapy with trastuzumab/pertuzumab in the neoadjuvant setting (Burstein et al., 2016), as it has been shown to increase pCR rates. According to the National Comprehensive Cancer Network (NCCN) Clinical Practice Guidelines in Oncology: Breast Cancer (NCCN, 2018a), the preferred regimens for neoadjuvant therapy for HER2+ BC are doxorubicin and cyclophosphamide followed by paclitaxel plus trastuzumab with or without pertuzumab, or docetaxel, carboplatin, and trastuzumab with or without pertuzumab. The NCCN Guidelines also list a host of other combination regimens that are appropriate neoadjuvant regimens.

Support for these recommendations is from the NeoSphere trial (Gianni et al., 2012), in which pertuzumab and trastuzumab were assessed as companion treatments for 12 weeks across four different preoperative regimens: (1) docetaxel plus trastuzumab (group A); (2) docetaxel plus pertu- zumab plus trastuzumab (group B); (3) pertuzumab and trastuzumab without chemotherapy (group C); and (4) docetaxel plus pertuzumab (group D). Following surgery, patients received fluorouracil at $600 \mathrm{mg} / \mathrm{m}^{2}$, epirubicin at $90 \mathrm{mg} / \mathrm{m}^{2}$, and cyclophosphamide at $600 \mathrm{mg} / \mathrm{m}^{2}$ (FEC) every 3 weeks for 3 cycles (group $\mathrm{C}$ received docetaxel for $4 \mathrm{cy}-$ cles prior to FEC), and trastuzumab at $6 \mathrm{mg} / \mathrm{kg} \mathrm{ev}$ ery 3 weeks to complete 1 year of treatment. A significantly higher proportion of patients achieved a pCR with combined pertuzumab and trastuzumab plus docetaxel than did those treated with trastuzumab plus docetaxel $(p=.014)$. The rate of pCR was higher in women with ER- tumors receiving pertuzumab and trastuzumab (27\%) compared with that in women with ER+ tumors receiving pertuzumab and trastuzumab (17\%).

A 5-year follow-up of NeoSphere (Gianni et al., 2016) showed that DFS and PFS were numerically higher in patients receiving docetaxel, trastuzumab, and pertuzumab for 12 weeks in the neoadjuvant setting than patients receiving docetaxel and trastuzumab. Five-year PFS rates were $81 \%(95 \%$ CI $=71 \%-87 \%)$ for docetaxel plus trastuzumab; $86 \%$ (95\% CI $=77 \%-91 \%)$ for docetaxel plus trastuzumab plus pertuzumab; 


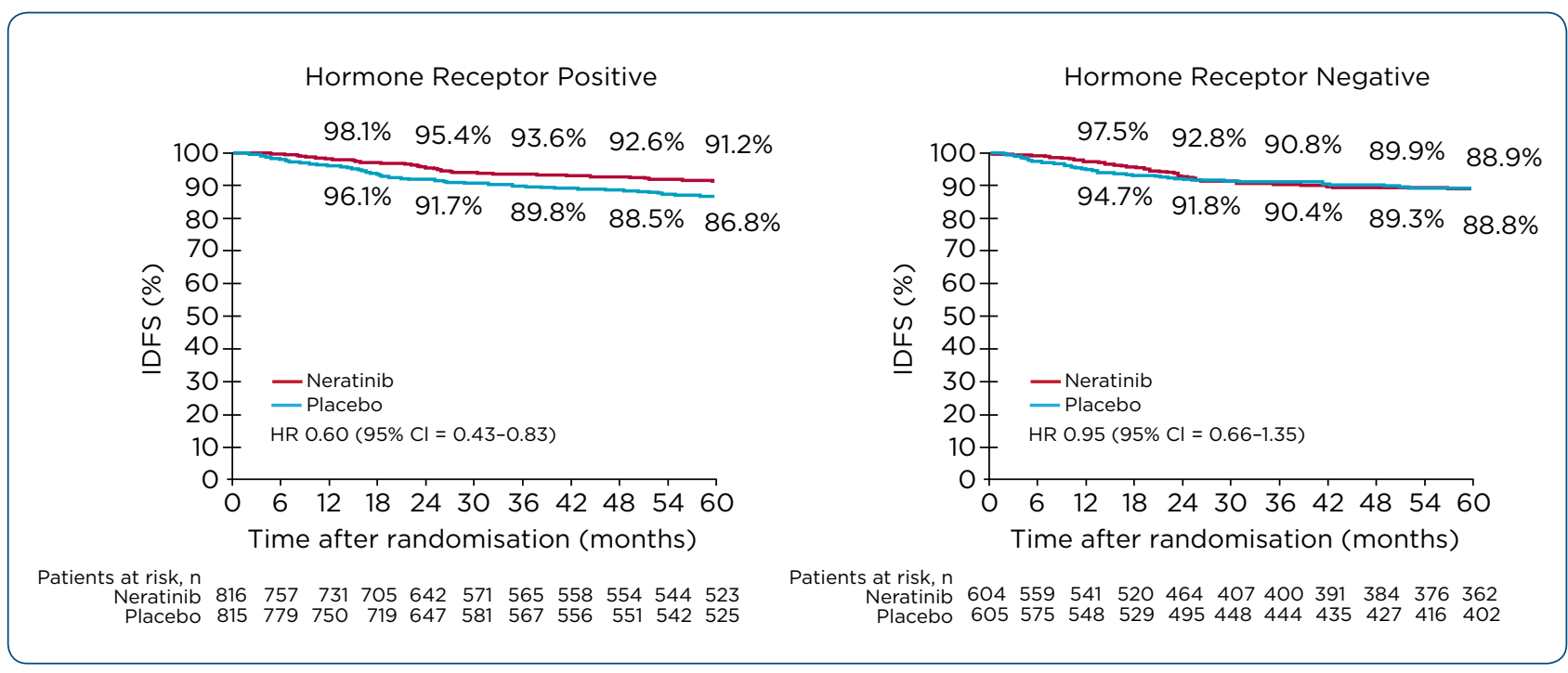

Figure 7. ExteNET: 5-year invasive disease-free survival analysis by hormone receptor status. Adapted from Martin et al. (2017).

$73 \%(95 \%$ CI $=64 \%-81 \%)$ for pertuzumab plus trastuzumab; and $73 \%$ (95\% CI $=63 \%-81 \%$ ) for docetaxel plus pertuzumab. Disease-free survival rates were nearly identical to PFS rates.

The TRYPHAENA trial (Schneeweiss et al., 2013) evaluated the efficacy of dual blockade of HER2 with trastuzumab and pertuzumab by combining pertuzumab with different anthracycline-containing and nonanthracycline-containing chemotherapy regimens. A total of 225 patients were randomized. The results confirmed that a high proportion of patients achieved a pCR with pertuzumab and trastuzumab as compared to trastuzumab alone (both in combination with chemotherapy).

\section{Case 2 Continued}

As mentioned above, the patient in case 2 did not achieve a pCR at the time of surgery, with residual disease identified in the breast. The standard of care for this patient is adjuvant trastuzumab emtansine (T-DM1), the benefit of which was established in the recently reported results of the KATHERINE trial.

\section{RESIDUAL DISEASE AFTER NEOADJUVANT CHEMOTHERAPY PLUS HER2 THERAPY: KATHERINE}

Patients with residual invasive BC after neoadjuvant chemotherapy plus HER2-targeted therapy have a worse prognosis than patients without residual disease.

In KATHERINE, patients with HER2+ early $\mathrm{BC}$ with residual invasive disease in the breast or axilla following surgery after the receipt of neoadjuvant therapy containing a taxane (with or without anthracycline) and trastuzumab, with $18 \%$ of patients also receiving pertuzumab, were randomly assigned to receive adjuvant T-DM1 or trastuzumab for 14 cycles (von Minckwitz et al., 2019). T-DM1 is an antibody-drug conjugate of trastuzumab and the cytotoxic agent emtansine (DM1), a maytansine derivative and microtubule inhibitor currently approved for HER2+ $\mathrm{MBC}$. At the interim analysis of 1,486 randomly assigned patients (743 in each group), invasive disease or death had occurred in 91 patients receiving T-DM1 (12.2\%), and 165 patients receiving trastuzumab (22.2\%). The estimated percentage of patients free of invasive disease at 3 years was $88.3 \%$ for T-DM1 and $77.0 \%$ for trastuzumab. Invasive DFS was significantly higher with T-DM1 than with trastuzumab (HR for invasive disease or death, 0.50; 95\% CI $=0.39-0.64 ; p<.001)$. Distant recurrence as the first invasive-disease event occurred in $10.5 \%$ of patients receiving T-DM1 and $15.9 \%$ receiving trastuzumab. Of note, even patients who had low volume residual disease demonstrated a benefit from receiving T-DM1 as compared to trastuzumab. 
Table 2. Summary of Adverse Events in the Safety Populationa

\begin{tabular}{|c|c|c|}
\hline Event, no. of patients (\%) & Trastuzumab group $(\mathrm{N}=720)$ & T-DM1 group $(N=740)$ \\
\hline Any adverse event & $672(93.3)$ & $731(98.8)$ \\
\hline Grade $\geq 3$ adverse event & $111(15.4)$ & $190(25.7)$ \\
\hline Adverse event leading to death ${ }^{b}$ & 0 & $1(0.1)$ \\
\hline Serious adverse event & $58(8.1)$ & $94(12.7)$ \\
\hline Adverse event leading to discontinuation of trial drug ${ }^{c}$ & $15(2.1)$ & $133(18.0)$ \\
\hline \multicolumn{3}{|l|}{$\begin{array}{l}\text { Grade } \geq 3 \text { adverse event that occurred in } \geq 1 \% \text { of patients in } \\
\text { either group }\end{array}$} \\
\hline Decreased platelet count & $2(0.3)$ & $42(5.7)$ \\
\hline Hypertension & $9(1.2)$ & $15(2.0)$ \\
\hline Radiation-related skin injury & $7(1.0)$ & $10(1.4)$ \\
\hline Peripheral sensory neuropathy & 0 & $10(1.4)$ \\
\hline Decreased neutrophil count & $5(0.7)$ & $9(1.2)$ \\
\hline Hypokalemia & $1(0.1)$ & $9(1.2)$ \\
\hline Fatigue & $1(0.1)$ & $8(1.1)$ \\
\hline Anemia & $1(0.1)$ & $8(1.1)$ \\
\hline \multicolumn{3}{|c|}{$\begin{array}{l}\text { Note. Adapted from von Minckwitz et al. (2019). } \\
\text { a Listed are adverse events with an onset that occurred from the first dose of any trial treatment through } 30 \text { days after } \\
\text { the final dose of trial treatment and adverse events with an onset in the follow-up period that were determined by the } \\
\text { investigators to be related to the trial drug or trial procedure. Patients may have had more than one adverse event. } \\
\text { b One patient with a platelet count of } 55,000 / \mathrm{mm}^{3} \text { fell at home and died of an intracranial hemorrhage. } \\
\text { 'The most common adverse event leading to discontinuation of the trial drug in the trastuzumab group was a } \\
\text { decreased ejection fraction in } 10 \text { of } 720 \text { patients }(1.4 \%) \text {. The most common adverse events leading to discontinuation } \\
\text { of the trial drug in the T-DM1 group were a decreased platelet count in } 31 \text { of } 740 \text { patients ( } 4.2 \%) \text {, an increased blood } \\
\text { bilirubin level in } 19 \text { patients ( } 2.6 \%) \text {, an increased aspartate aminotransferase level in } 12 \text { patients }(1.6 \%) \text {, an increased } \\
\text { alanine aminotransferase level in } 11 \text { patients (1.5\%), peripheral sensory neuropathy in } 11 \text { patients (1.5\%), and a decreased } \\
\text { ejection fraction in } 9 \text { patients }(1.2 \%) \text {. }\end{array}$} \\
\hline
\end{tabular}

A total of 740 patients in the T-DM1 group and 720 in the trastuzumab group were included in the safety analysis, as shown in Table 2.

The most common AEs grade 3 or higher were decreased platelet counts and hypertension in the T-DM1 group and hypertension and radiation-related skin injury in the trastuzumab group. Serious AEs occurred in $12.7 \%$ of patients receiving T-DM1 and $8.1 \%$ of patients receiving trastuzumab. Adverse event-related discontinuation occurred in $18 \%$ of patients receiving T-DM1 and $2.1 \%$ receiving trastuzumab. Adverse events of any grade were slightly more common in the T-DM1 group. In addition to the safety data presented in Table 2, AEs of any grade with an incidence of at least 15\% were noted (von Minckwitz et al., 2018), with fatigue presenting in about one third of patients in the trastuzumab group and nearly $50 \%$ of the patients receiving T-DM1. The authors note that the safety profile for T-DM1 was consistent with that of previous studies and, as expected, there were more AEs in patients receiving T-DM1 than in those receiving adjuvant trastuzumab.

Although the ExteNET trial as described above did not include patients who had received adjuvant T-DM1, in this situation, it would be reasonable to consider extended adjuvant neratinib, but there are as yet no data supporting this approach.

\section{Key Points}

- Patients with residual disease after neoadjuvant chemotherapy plus HER2 targeted therapy have a worse prognosis than do those without residual disease

- A significantly higher proportion of patients achieve a pCR with combined pertuzumab and trastuzumab plus docetaxel than do those treated with trastuzumab plus docetaxel 
- DFS and PFS are higher in patients receiving docetaxel, trastuzumab, and pertuzumab for 12 weeks in the neoadjuvant setting than in patients receiving docetaxel and trastuzumab

- Even patients with very low volume residual disease demonstrate a benefit from T-DM1 as compared to trastuzumab

\section{Case 3: Strategies for Managing AEs}

The patient is a 60-year-old woman with no prior relevant medical history. A mass was palpated in her left breast, biopsy-proven to be invasive ductal carcinoma, HER2+ (3+ by IHC), ER+. The tumor was approximately $2.5 \mathrm{~cm}$ by imaging with suspicious axillary nodes that were biopsy-proven to be involved, cT2N1M0. She received neoadjuvant therapy with TCHP $\times 6$ cycles and has significant side effects from therapy, including diarrhea, rash, and general intolerance. The patient was able to complete the 6 cycles of therapy but had residual disease at the time of surgery. She was offered trastuzumab emtansine (T-DM1) in the adjuvant setting yet refused. She also refused pertuzumab and/or neratinib for fear of further side effects. She did consent to complete a year of trastuzumab in the adjuvant setting, but at 6 months into the planned 1 year of treatment, she developed severe headaches and was found to have brain metastases.

\section{ADVERSE EVENT MANAGEMENT}

To date, there is substantial experience with $\mathrm{AE}$ management to help patients who receive trastuzumab, pertuzumab and/or neratinib for the management of HER2+ BC. Diarrhea and rash are common side effects of pertuzumab. Both trastuzumab and pertuzumab can be associated with cardiac toxicity and infusion-related events (albeit rarely). Early identification and management of side effects is recommended.

For neratinib, diarrhea is the main side effect. It can be severe in patients who do not receive antidiarrheal prophylaxis. Other toxicities include liver toxicity (rare) and a variety of drug interactions related to gastric-acid-reducing agents, CYP3A4 inhibitors/inducers, and P-glycoprotein substrates.

As demonstrated in the CONTROL trial, antidiarrhea prophylaxis with the use of loperamide, budesonide, and colestipol reduces the incidence, severity, and duration of neratinib-associated di- arrhea (Martin et al., 2017). In addition to this approach, other measures to decrease the incidence of diarrhea and prevent dehydration include instructing patients to remain hydrated by drinking 8 to 10 glasses of water daily, and having patients avoid high-fat greasy, spicy, or fried foods.

In terms of diet management, patients should eat small but frequent meals and consider foods low in fiber, high in protein and potassium, and containing live bacterial cultures (such as yogurt) or probiotics. Patients should avoid dairy, alcohol and caffeine, high-fiber foods, and foods containing grapefruit (Benson et al., 2004).

The advanced practitioner's role in managing patients on HER2 therapy overall, inclusive of addressing diarrhea, is best encapsulated as follows:

- Essential baseline assessments

» Extent of disease

» Cardiovascular history and subjective assessments (LVEF)

" Gastrointestinal history and subjective assessments as needed

- Patient education

»Disease process

» Treatment

»Self-management of potential toxicities that is proactive, not reactive

" Prescribing antidiarrhea medications to have on hand with specific written instructions for when to start and what to report (i.e., loperamide $4 \mathrm{mg}$ po at first episode of diarrhea and every 4-6 hours as needed; do not exceed $16 \mathrm{mg}$ per day)

» Coordinate office personnel to contact patient on days $1,8,15$, and 22 of first month of therapy to discuss if diarrhea is a problem or well controlled if patient is not being seen at that time for office visits

»Encourage patient that diarrhea usually starts to taper towards normal after first 1-2 months

» If diarrhea is uncontrolled with loperamide, consider adding prescription atropine/diphenoxylate 2.5 -mg tablets every 4-6 hours, not to exceed 8 tablets per day

" May add colestipol $2 \mathrm{mg}$ bid 4 hours before or 2 hours after neratinib first cycle

»Discuss diet restrictions: low-residue foods, bananas, rice, bread, mashed pota- 
toes, yogurt, probiotics, chicken broth, no fried/greasy/spicy foods, cream of wheat

»Call office if maximum dose ineffective

" Report AEs: when and to whom

» Encourage increased oral fluid intake with water and electrolyte-replacing drinks (Gatorade or Pedialyte if appropriate)

"Schedule for electrolyte assessment and replace fluids through IV hydration if oral not possible

- Staff education

Taking baseline measurements is essential to assessing changes that are treatment-related. Educating the patient on what to expect and how to address AEs preventively is also critical, as is educating any staff who interface with the patient (NCCN, 2017).

For this particular patient, it is critical to treat her diarrhea with hydration and diet, more so if it is sufficiently severe, using loperamide or diphenoxylate after each episode until it subsides. Rash that is not severe may not affect the completion of the current cycle of treatment, but treatment with minocycline or a corticosteroid may be required.

\section{AGENTS IN DEVELOPMENT, INCLUDING THE UNMET NEED OF TARGETED THERAPIES FOR BRAIN METASTASES IN HER2+ MBC}

Although considerable progress has been made in the treatment of HER2+ MBC there is still a need for additional treatments for these patients. Standard first-line therapy in the metastatic setting is a taxane, trastuzumab, and pertuzumab based on the CLEOPATRA trial (Swain et al., 2015). T-DM1 is often used as second-line therapy based on the EMILIA trial (Diéras et al., 2017). However, for third-line therapy and beyond, several new agents are under investigation. One new agent is margetuximab (Burris et al., 2015; Rugo et al., 2016), which expresses an enhanced antibody-dependent cellular cytotoxicity leading to tumor cell destruction.

Margetuximab is being evaluated in SOPHIA, a phase III trial comparing a chemotherapy backbone plus margetuximab vs. chemotherapy plus trastuzumab in the treatment of HER2+ MBC. On February 6, 2019, positive results from this trial were released (MacroGenetics, 2019). Patients in the margetuximab arm experienced a $24 \%$ risk reduction of disease progression or death vs. patients in the trastuzumab arm (HR, 0.76; $p=.033)$. Of note, approximately $85 \%$ of patients in SOPHIA were CD16A (FcyRIIIa) 158F allele carriers, previously linked to a diminished clinical response to trastuzumab and other antibodies. For this margetuximab arm subgroup there was a $32 \%$ risk reduction of disease progression or death vs. trastuzumab (HR, 0.68; $p=.005)$. These findings are noteworthy as there are currently no approved agents for the treatment of patients with metastatic HER2+ BC who have previously received trastuzumab, pertuzumab, and TDM1.

Breast cancer metastatic to the brain continues to be a troubling occurrence for many women (Frisk et al., 2017; Jin et al., 2018; Leone \& Leone, 2015; Shen et al., 2015). While there has been little to offer these patients, there have been recent advances and an appreciation of new strategies to address this unmet need. In regard to brain metastases, promising results have been reported with the use of tucatinib, also known as ONT-380. Tucatinib has a high selectivity for HER2 $\left(\mathrm{IC}_{50(\mathrm{nM})}=8\right.$ for HER2; $\mathrm{IC}_{50(\mathrm{nM})}=>10,000$ for EGFR; Moulder et al., 2017).

When comparing cellular selectivity to HER2 or to EGFR, these data suggest that tucatinib may have less skin and GI toxicity as compared to other antiHER2 agents. In a phase $1 \mathrm{~b}$ study when combined with capecitabine with our without trastuzumab in women with HER2+ MBC, the objective response rate was $61 \%$ with a median PFS of nearly 8 months. In June 2017, tucatinib was given an orphan drug designation by the FDA for the treatment of BC with brain metastases (Cascadian Therapeutics, 2017). A phase II trial of tucatinib plus capecitabine plus placebo vs. tucatinib in combination with capecitabine and trastuzumab in HER2 + BC is underway, the primary endpoint being PFS with secondary endpoints of overall response rate, duration of response, clinical benefit response, safety, and tolerability (NCT02614794; ClinicalTrials.gov, 2019).

Another option for patients with HER2+ MBC with brain metastases is neratinib plus capecitabine, which is listed as a Category 2A recommendation treatment option by NCCN (in the central nervous system section of the NCCN Guidelines; 2018b). The recommendation was based on a phase II trial, the TBCRC 022 study. In 
addition, there is a Category $2 \mathrm{~B}$ recommendation for neratinib plus paclitaxel for this same group of patients. The ongoing NALA trial is a phase III randomized controlled trial of neratinib plus capecitabine vs. lapatinib plus capectabine in patients with third-line HER2+ MBC. A press release in December 2018 indicated this was a positive trial with further results expected shortly (Puma Biotechnology, Inc., 2018).

\section{Key Points}

- APs should educate patients treated with neratinib to monitor for and prevent diarrhea; dietary precautions and treatment with loperamide and colestipol are helpful in reducing frequency and severity

- New approaches for patients with brain metastases are on the horizon

- Consider enrollment in clinical trials for patients with HER2+ MBC and central nervous system metastases with the option of neratinib plus capecitabine, to be considered per NCCN Guidelines

\section{Case 4: Therapy De-Escalation and Cardiac Toxicity}

The final case features a 65-year-old woman with a history of obesity, hypertension, high cholesterol, borderline diabetes mellitus, and prior myocardial infarction, presenting with $1.5 \mathrm{~cm}$, nodenegative, ER+/HER2+ BC. She had a lumpectomy and then received weekly paclitaxel/trastuzumab (paclitaxel weekly $\times 12$ weeks with trastuzumab to complete 1 year). She received post-lumpectomy radiation and was started on adjuvant hormonal therapy. Six months into the year of trastuzumab, she developed a symptomatic drop in ejection fraction that did not resolve by withholding the drug or with the use of cardiac medications as recommended by her cardiologist.

\section{THERAPY DE-ESCALATION AND CARDIAC TOXICITY}

Data supporting de-escalation of therapy as administered in this case is found in the APT trial by Tolaney and colleagues. This was a singlearm, phase II nonrandomized trial to evaluate if 12 weeks of adjuvant paclitaxel in combination with trastuzumab was effective in node-negative, small $(<3 \mathrm{~cm})$, HER2 + BC. A total of 410 patients were enrolled. The overall 7-year DFS was 93.3\% and the overall 7-year relapse-free interval was $97.5 \%$. It is important to note that two-thirds of patients had HR-positive tumors, and recurrences in this subset may be seen well after 3 years (Tolaney et al., 2015, 2017).

This case also highlights the studies that have evaluated shorter durations (less than 1 year) of adjuvant trastuzumab. Multiple studies have failed to demonstrate noninferiority of shorter durations of trastuzumab, and the HERA trial failed to demonstrate the superiority of 2 years of trastuzumab therapy as compared to 1 year of trastuzumab therapy.

The only study demonstrating noninferiority was the recently presented PERSEPHONE study (Earl et al., 2018), in which patients were given chemotherapy $\times 6$ cycles and then given 6 months or 1 year of trastuzumab concurrently/sequentially with chemotherapy. Although the noninferiority endpoint was met, several caveats from the study are worth mention. This trial included a fairly good-risk population, with $70 \%$ of patients having $\mathrm{ER}+$ disease and $30 \%$ of patients having ER- disease (Earl et al., 2018). Few patients today receive only anthracycline-based or taxane-based therapy, but in PERSEPHONE some did, and a few received cyclophosphamide, methotrexate, and fluorouracil, which is not standard therapy for patients with HER $2+$ BC. Approximately $60 \%$ of patients were node-negative and $40 \%$ were node-positive. In 2018, an updated analysis at a median follow-up at 5.4 years was presented where non-inferiority to a shorter duration of adjuvant trastuzumab (6 months vs. 1 year) was met (Earl et al., 2018). Subset analyses indicated the longer duration of trastuzumab was superior in patients with ER- disease than in those patients who received sequential as opposed to concurrent trastuzumab therapy (Earl et al., 2018). Therefore, results of this trial should not be broadly generalized, and 1 year of adjuvant trastuzumab remains the standard of care. However, for patients such as the one described in case 4 , shorter durations of therapy may be considered based on these results.

\section{Key Points}

- Although 1 year of adjuvant trastuzumab is the standard of care, a shorter duration 
of therapy may be considered for some patients based on their unique characteristics and disease history; multiple studies have failed to demonstrate the noninferiority of shorter durations of trastuzumab

- It is important to obtain cardiac echo or MUGA scans every 3 months to monitor for potential cardiotoxicity for patients receiving trastuzumab (alone or in combination with pertuzumab) and to appropriately hold therapy as per guidelines.

\section{CONCLUSIONS}

The discovery of the HER2 gene and HER2 targeted therapies has resulted in decreases in $\mathrm{BC}$ associated morbidity and mortality. However, patients with HER2+ BC remain at risk for recurrent disease, which is not considered curable and is associated with a decrease in overall survival. Therefore, there is room to further improve outcomes for early-stage HER2+ BC patients who receive treatment in the adjuvant setting and neoadjuvant settings.

Several data sets highlighted in this article support the consideration of neoadjuvant chemotherapy with trastuzumab and pertuzumab in patients with node-positive disease and/or tumors of $2 \mathrm{~cm}$ or greater in size. For patients with residual disease following neoadjuvant therapy, the KATHERINE trial supports a new standard of care for the use of T-DM1 in the adjuvant setting. Based on results from the APHINITY and the ExteNET trials, there is now data to support the addition of concurrent pertuzumab for high-risk patients in the adjuvant setting or the sequential use of neratinib in $\mathrm{HR}+$ patients after a year of trastuzumab in the extended adjuvant setting.

In the metastatic setting there are several novel agents under investigation that appear promising, and a focus on developing therapies for patients with brain metastases, as this is an area of high unmet need. It is important to be mindful of therapy-associated toxicities of all these treatments and to institute early management strategies to adequately support patients through treatment. As treatment continues to be refined, future efforts should remain focused on identifying biomarkers for improving patient selection in order to successfully exclude those who are unlikely to benefit from these expensive interventions.

\section{CLINICAL PEARLS}

To optimize care for patients with HER2+ BC, APs should:

- Stay abreast of emerging data regarding current and novel neoadjuvant, adjuvant, extended adjuvant and metastatic therapies; discuss the clinical implications with their care team to ensure evolving data can be safely and effectively integrated into practice

- Be proactive in monitoring for and treating AEs and encourage patients and their caregivers to do so as well

- Educate patients treated with neratinib to remain hydrated; avoid high-fat greasy, spicy, or fried foods; and take medication to prevent diarrhea

- It is important to obtain cardiac echo or MUGA scans every 3 months to monitor for potential cardiotoxicity for patients receiving trastuzumab (alone or in combination with pertuzumab) and to appropriately hold therapy as per guidelines.

- Be aware of the higher risk of brain metastases in HER2+ MBC and recognize there are agents under investigation for this area of high unmet need.

\section{Disclosure}

Dr. Mahtani has consulted for Amgen, Genentech, Lilly, Novartis, Pfizer, and Puma, and has received research support from Genentech. Ms. Hineman has nothing to disclose.

\section{References}

American Cancer Society. (2018). Cancer Facts \& Figures 2018. Atlanta, GA: American Cancer Society.

Benson, A. B., 3rd, Ajani, J. A., Catalano, R. B. Engelking, C., Kornblau, S. M., Martenson, J. A., Jr.,...Wadler, S. (2004). Recommended guidelines for the treatment of cancer treatment-induced diarrhea. Journal of Clinical Oncology, 22(14), 2918-2926. https://doi.org/10.1200/ JCO.2004.04.132

Burris, H. A., Giaccone, G., Im, S.-A., Bauer, T. M., Oh, D.-.Y, Jones, S. F.,...Bang, Y.-J. (2015). Updated findings of a first-in-human, phase I study of margetuximab (M), an Fc-optimized chimeric monoclonal antibody (MAb), in patients (pts) with HER2-positive advanced solid tumors [Abstract 523]. Journal of Clinical Oncology (ASCO Annual Meeting Abstracts), 33(15 suppl). https://doi. 
org/10.1200/jco.2015.33.15_suppl.523

Burstein, H. J. (2005). The distinctive nature of HER2-positive breast cancers. New England Journal Medicine, 353(16), 1652-1654. https://doi.org/10.1056/NEJMp058197

Burstein, H. J., Lacchetti, C., Anderson, H., Buchholz, T. A., Davidson, N. E., Gelmon, K. E.,...Griggs, J. J. (2016). Adjuvant endocrine therapy for women with hormone receptor-positive breast cancer: American Society of Clinical Oncology Clinical Practice Guideline Update on Ovarian Suppression. Journal of Clinical Oncology, 34(14), 16891701. https://doi.org/10.1200/JCO.2015.65.9573

Cascadian Therapeutics. (2017). FDA grants orphan drug designation to tucatinib for brain metastases. Retrieved from https://www.healio.com/hematology-oncology/ breast-cancer/news/online/\%7Bbdcef13f-c984-41959a4b-ef06fcdd $8510 \% 7 \mathrm{D} /$ fda-grants-orphan-drug-designation-to-tucatinib-for-brain-metastases

Chan, A., Delaloge, S., Holmes, F. A., Moy, B., Iwata, H., Harvey, V. J.,...Martin, M. (2016). Neratinib after trastuzumabbased adjuvant therapy in patients with HER2-positive breast cancer (ExteNET): A multicentre, randomised, double-blind, placebo-controlled, phase 3 trial. Lancet Oncology, 17(3), 367-377, https://doi.org/10.1016/S14702045(15)00551-3

ClinicalTrials.gov. (2019). A study of tucatinib vs. placebo in combination with capecitabine \& trastuzumab in patients with advanced HER2+ breast cancer (HER2CLIMB). Retrieved from https://clinicaltrials.gov/ct2/ show/NCT02614794

Diéras, V., Miles, D., Verma, S., Pegram, M., Welslau, M., Baselga, J.,...Gianni, L. (2017). Trastuzumab emtansine versus capecitabine plus lapatinib in patients with previously treated HER2-positive advanced breast cancer (EMILIA): A descriptive analysis of final overall survival results from a randomised, open-label, phase 3 trial. Lancet Oncology, 18(6), 732-742. https://doi.org/10.1016/ S1470-2045(17)30312-1

Earl, H. M., Hiller, L., Vallier, A. L., Loi, S., Howe, D., Higgins, H. B.,...Dunn, J. (2018). PERSEPHONE: 6 versus 12 months of adjuvant trastuzumab in patients with HER2positive breast cancer [Abstract 506]. Journal of Clinical Oncology (ASCO Annual Meeting Abstracts), 36(suppl). Retrieved from https://meetinglibrary.asco.org/record/160257/abstract

Frisk, G., Tinge, B., Ekberg, S., Eloranta, S., Bäcklund, M., Lidbrink, E., \& Smedby, K. E. (2017). Survival and level of care among breast cancer patients with brain metastases treated with whole brain radiotherapy. Breast Cancer Research and Treatment, 166, 887-896. https://doi. org/10.1007/s10549-017-4466-3

Genentech, Inc. (2012). Perjeta (pertuzumab) package insert. Retrieved from https://www.accessdata.fda.gov/drugsatfda_docs/label/2012/125409lbl.pdf

Gianni, L., Pienkowski, T., Im, Y.-H., Roman, L., Tseng, L.M., Liu, M.-C.,...Valagussa, P. (2012). Efficacy and safety of neoadjuvant pertuzumab and trastuzumab in women with locally advanced, inflammatory, or early HER2positive breast cancer (NeoSphere): A randomised multicentre, open-label, phase 2 trial. Lancet Oncology, 13(1), 25-32. https://doi.org/10.1016/S1470-2045(11)70336-9

Gianni, L., Pienkowski ,T., Im, Y.-H., Tseng, L.M., Liu, M.C., Lluch, A.,...Valagussa, P. (2016). 5-year analysis of neoadjuvant pertuzumab and trastuzumab in patients with locally advanced, inflammatory, or early-stage HER2positive breast cancer (NeoSphere): A multicentre, openlabel, phase 2 randomised trial. Lancet Oncology, 17(6), 791-800. https://doi.org/10.1016/S1470-2045(16)00163-7

Goldhirsch, A., Gelber, R. D., Piccart-Gebhart, M. J., de Azambuja, E., Procter, M., Suter, T. M.,...Baselga, J. (2013). 2 years versus 1 year of adjuvant trastuzumab for HER2positive breast cancer (HERA): An open-label, randomised controlled trial. Lancet, 382(9897), 1021-1028. https://doi.org/10.1016/S0140-6736(13)61094-6

Howlader, N., Altekruse, S. F., Li, C. I., Chen, V. W., Clarke, C. A., Ries, L. A. G., \& Cronin, K. A. (2014). US incidence of breast cancer subtypes defined by joint hormone receptor and HER2 status. Journal of the National Cancer Institute, 106(5), dju055. https://doi.org/10.1093/jnci/ dju055

Hurvitz, S., Chan, A., Iannotti, N., Ibrahin, E., Chien, J., Chan, N.,...Rugo, H. S. (2017). Abstract P3-14-01: Effects of adding budesonide or colestipol to loperamide prophylaxis on neratinib-associated diarrhea in patients with HER2+ early-stage breast cancer: The CONTROL trial [Abstract P3-14-01]. Presented at the San Antonio Breast Cancer Symposium. Retrieved from https://www.sabcs.org/Portals/SABCS2016/Documents/SABCS-2017-Abstracts.pdf

Jin, J., Gao, Y., Wang, L., Wang, B., Cao, J., Shao, Z., \& Wang, $Z$. (2018). Incidence, pattern and prognosis of brain metastases in patients with metastatic triple negative breast cancer. BMC Cancer, 18, 446. https://doi.org/10.1186/ s12885-018-4371-0

Leone, J. P., \& Leone, B. A. (2015). Breast cancer brain metastases: The last frontier. Experimental Hematology $\mathcal{E}$ Oncology, 4, 33. https://doi.org/10.1186/s40164-015-0028-8

Martin, M., Holmes, F. A., Ejlertsen, B., Delaloge, S., Moy, B., Iwata, H.,...Chan, A. (2017). Neratinib after trastuzumabbased adjuvant therapy in HER2-positive breast cancer (ExteNET): 5-year analysis of a randomised, doubleblind, placebo-controlled, phase 3 trial. Lancet Oncology, 18(12), 1688-1700. https://doi.org/10.1016/S14702045(17)30717-30719

Moulder, S. L., Borges, V. F., Baetz, T., Mcspadden, T., Fernetich, G., Murthy, R. K.,...Chia, S. K. (2017). Phase I study of ONT-380, a HER2 inhibitor, in patients with HER2+-advanced solid tumors, with an expansion cohort in HER2+ metastatic breast cancer (MBC). Clinical Cancer Research, 23(14), 3529-3536. https://doi. org/10.1158/1078-0432.CCR-16-1496

National Comprehensive Cancer Network. (2017). NCCN Clinical Practice Guidelines in Oncology: Breast Cancer. V2.2017. Retrieved from https://jnccn.org/abstract/journals/jnccn/15/6/article-p804.xml

National Comprehensive Cancer Network. (2018). NCCN Clinical Practice Guidelines in Oncology: Breast Cancer. V3.2018. Retrieved from https://www.nccn.org/professionals/physician_gls/pdf/breast.pdf

National Comprehensive Cancer Network. (2018). NCCN Clinical Practice Guidelines in Oncology: Central nervous system cancers. V2.2018. Retrieved from https:// www.nccn.org/professionals/physician_gls/pdf/cns.pdf

Perez, E. A., Romond, E. H., Suman, V. J., Jeong, J. H., Sledge, G., Geyer, C. E., Jr.,...Wolmark, N. (2014). Trastuzumab plus adjuvant chemotherapy for human epidermal growth factor receptor 2-positive breast cancer: Planned joint analysis of overall survival from NSABP B-31 and 
NCCTG N9831. Journal of Clinical Oncology, 32(33), 3744-3752. https://doi.org/10.1200/JCO.2014.55.5730

Puma Biotechnology, Inc. (2017). Nerlynx (neratinib) package insert. Retrieved from https://www.accessdata.fda. gov/drugsatfda_docs/label/2017/208051s000lbl.pdf

Puma Biotechnology, Inc. (2018). Puma biotechnology announces top line results of the phase III NALA trial of neratinib in patients with HER2-positive metastatic breast cancer. Retrieved from https://investor. pumabiotechnology.com/press-release/puma-biotechnology-announces-top-line-results-phase-iii-nalatrial-neratinib-patients-

Romond, E. H., Perez, E. A., Bryant, J., Suman, V. J., Geyer, C. E., Jr., Davidson, N. E.,...Wolmark, N. (2005). Trastuzumab plus adjuvant chemotherapy for operable HER2-positive breast cancer. New England Journal of Medicine, 353(16), 1673-1684. https://doi.org/10.1056/ NEJMoa052122

Rugo, H. S., Pegram, M. D., Gradishar, W. J., Cortes, J., Curigliano, G., Wigginton, J. M.,...Cardoso, F. (2016). SOPHIA: A phase 3, randomized study of margetuximab plus chemotherapy (CTX) vs trastuzumab plus CTX in the treatment of patients with HER2+ metastatic breast cancer [Abstract TPS630]. Journal of Clinical Oncology (ASCO Annual Meeting Abstracts), 34(suppl 15). Retrieved from https://meetinglibrary.asco.org/record/126836/abstract

Schneeweiss, A., Chia, S., Hickish, T., Harvey, V., Eniu A., Hegg, R,...Cortez, J. (2013). Pertuzumab plus trastuzum$\mathrm{ab}$ in combination with standard neoadjuvant anthracycline-containing and anthracycline-free chemotherapy regimens in patients with HER2-positive early breast cancer: A randomized phase II cardiac safety study (TRYPHAENA). Annals of Oncology, 24(9), 2278-2284, https://doi.org/10.1093/annonc/mdt182

Shen, Q., Sahin, A. A., Hess, K. R., Suki, D., Aldape, K. D., Sawaya, R., \& Ibrahim, N. K. (2015). Breast cancer with brain metastases: Clinicopathologic features, survival, and paired biomarker analysis. Oncologist, 20(5), 466473. https://doi.org/10.1634/theoncologist.2014-0107

Slamon, D., Eiermann, W., Robert, N., Pienkowski, T., Martin, M., Press, M.,...Crown, J. (2011). Adjuvant trastuzumab in HER2-positive breast cancer. New England Journal of Medicine, 365(14), 1273-1283. https://doi.org/10.1056/ NEJMoa0910383

Slamon, D. J., Eiermann, W., Robert, N. J., Giermek, J., Martin, M., Jasiowka, M.,...Crown, J. (2015). Ten year follow-up of BCIRG-006 comparing doxorubicin plus cyclophosphamide followed by docetaxel with doxorubicin plus cyclophosphamide followed by docetaxel and trastuzumab with docetaxel, carboplatin, and trastuzumab in HER2-positive early breast cancer [Abstract S5-04]. Presented at the San Antonio Breast Cancer Symposium. Retrieved from https://www.abstracts2view.com/sabcs15/ view.php?nu=SABCS15L_1030\&terms=

Swain, S. M., Baselga, J., Kim, S.-B., Ro, J., Semiglazov, V., Campone, M.,...Cortés, J. (2015). Pertuzumab, trastuzumab, and docetaxel in HER2-positive metastatic breast cancer. New England Journal Medicine, 372(8), 724-734. https://doi.org/10.1056/NEJMoal413513

Tolaney, S. M., Barry, W. T., Dang, C. T., Yardley, D. A., Moy, B., Marcom, P. K.,...Winer, E. P. (2015). Adjuvant paclitaxel and trastuzumab for node-negative, HER2-positive breast cancer. New England Journal of Medicine, 372(2), 134-141. https://doi.org/10.1056/NEJMoa1406281

Tolaney, S. M., Barry, W. T., Guo, H., Dillon, D, Dang, C. T., Yardley, D. A.,...Winer, E. A. (2017). Seven-year follow-up of adjuvant paclitaxel and trastuzumab (APT trial) for node-negative, HER2-positive breast cancer [Abstract 511]. Journal of Clinical Oncology (ASCO Annual Meeting Abstracts), 35(suppl). Retrieved from https://meetinglibrary.asco.org/record/145548/abstract

von Minckwitz, G., Huang, C. S., Mano, M. S., Loibl, S., Mamounas, E. P., Untch, M.,...Geyer, C. E. (2019). Trastuzumab emtansine for residual invasive HER2-positive breast cancer. New England Journal of Medicine, 380, 617-628. https://doi.org/10.1056/NEJMoa1814017

von Minckwitz, G., Procter, M., de Azambuja, E., Zardavas, D., Benyunes, M., Viale, G.,...Baselga, J. (2017). Adjuvant pertuzumab and trastuzumab in early HER2-positive breast cancer. New England Journal of Medicine, 377(2), 122-131. https://doi.org/10.1056/NEJMoa1703643

\section{How to Earn Credit}

To access the learning assessment and evaluation form online, visit https://education.annenberg.net/jadpro-her2article

Statement of Credit: Participants who successfully complete this activity (including scoring of a minimum of $70 \%$ on the learning assessment) and complete and submit the evaluation form will be able to download a statement of credit. 